PALEO

Revue d'archéologie préhistorique

$14 \mid 2002$

Varia

\title{
Présence de Panthera Gombaszoegensis Kretzoï, 1938 à la grotte XIV (Cénac-et-Saint-Julien, Dordogne)
}

Presence of Panthera gombaszoegensis Kretzoï, 1938 in Grotte XIV (Cénac et Saint-Julien, Dordogne)

\section{Anne Langlois}

\section{OpenEdition}

\section{Journals}

Édition électronique

URL : http://journals.openedition.org/paleo/1449

DOI : $10.4000 /$ paleo.1449

ISSN : 2101-0420

Éditeur

SAMRA

Édition imprimée

Date de publication : 1 décembre 2002

Pagination : 213-220

ISSN : $1145-3370$

Référence électronique

Anne Langlois, « Présence de Panthera Gombaszoegensis Kretzoï, 1938 à la grotte xIv (Cénac-et-SaintJulien, Dordogne) », PALEO [En ligne], 14 | 2002, mis en ligne le 17 août 2010, consulté le 27 juillet 2020. URL : http://journals.openedition.org/paleo/1449; DOI : https://doi.org/10.4000/paleo.1449

Ce document a été généré automatiquement le 27 juillet 2020.

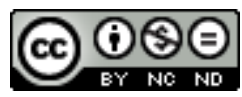

PALEO est mis à disposition selon les termes de la licence Creative Commons Attribution - Pas d'Utilisation Commerciale - Pas de Modification 4.0 International. 


\section{Présence de Panthera}

\section{Gombaszoegensis Kretzoï, 1938 à la grotte XIV (Cénac-et-Saint-Julien, Dordogne)}

Presence of Panthera gombaszoegensis Kretzoï, 1938 in Grotte XIV (Cénac et

Saint-Julien, Dordogne)

\section{Anne Langlois}

Je voudrais, tout d'abord, remercier J.-Ph. Rigaud, Directeur de l'Institut de Préhistoire et de Géologie du Quaternaire pour m'avoir accueillie dans ses locaux et permis de faire cette étude. Merci également à J.-F. Tournepiche pour m'avoir permis d'observer le félin d'Artenac ainsi que pour l'accueil sur le site lui-même qu'il dirige avec A. Delagnes.

Je n'oublie pas non plus J.-L. Guadelli pour ses conseils et son aide pour ce travail mais aussi pour les étés de fouilles à la Grotte XIV.

1 Panthera gombaszoegensis, grand félin fossile dont la taille se situe entre celle du Jaguar et celle du Lion actuel, vivait au Pléistocène ancien (Villafranchien supérieur : -1,8 à -1 million d'années) et au Pléistocène moyen jusque vers 350000 ans. C'est une espèce ubiquiste. A. Argant (1989) indique que dans le gisement de Château, suivant les données palynologiques (Argant J. 1980), sa présence donne des “ indications ponctuelles d'une période tempérée plus froide que l'actuelle et d'une autre plus froide encore. Les arbres étaient présents (..., mais) le milieu ouvert existait aussi. Il n'est donc pas possible de considérer Panthera gombaszoegensis comme uniquement forestier " (Argant 1989, p. 221).

2 Panthera gombaszoegensis a été identifiée par M. Kretzoï en 1938 à partir des restes découverts à Gombaszög, en Hongrie (Pléistocène ancien ; Villafranchien supérieur : 1,8 à -1 million d'années) et la diagnose repose sur des dents isolées (carnassière supérieure, $\mathrm{P} / 3, \mathrm{P} / 4, \mathrm{M} / 1$, canines). D'après $\mathrm{M}$. Kretzoï, la taille de Panthera gombaszoegensis se situerait entre celle du Jaguar (Panthera onca) et celle du Lion 
(Panthera (Leo) leo). Certains auteurs le considèrent comme un Jaguar européen qui aurait vécu dans des zones boisées avec des cours d'eau (Guérin et Patou-Mathis 1996). La longueur du corps, tête comprise, pouvait atteindre de 120 à $170 \mathrm{~cm}$ et son poids pouvait varier de 60 à $160 \mathrm{~kg}$.

D'après B. Kurtén (1973), Panthera gombaszoegensis, forme proche du Jaguar, et Panthera onca, le Jaguar lui même, sont issus d'une même population qui vivait dans les régions circumpolaires au Pléistocène ancien. Quant à $\mathrm{H}$. Hemmer, il considère que "the European jaguars from the Upper Villafranchian and from the Epivillafranchian to lower Middle Pleistocene should be referred to as P. onca toscana and P. onca gombaszoegensis, respectively" ${ }^{1}$ (Hemmer et al. 2001, p. 476). Les jaguars d'Amérique du Nord devant, quant à eux, être rapportés à P. onca augusta.

En France, de plus en plus de fossiles sont découverts, décrits et attribués à Panthera gombaszoegensis: quatre sites archéologiques ont livré des restes en quantité non négligeable; il s'agit de Château en Saône-et-Loire, de la grotte de l'Escale dans les Bouches-du-Rhône, d'Artenac en Charente et de la Grotte XIV en Dordogne.

\section{Château (Saône-et-Loire)}

5 En Bourgogne, à Château, A. Argant (1989) a découvert des fragments de maxillaire, de mandibule, des dents isolées $(\mathrm{I} / 2, \mathrm{P} / 3, \mathrm{P} / 4, \mathrm{M} / 1)$ et des fragments de squelette postcrânien. Il rapporte ces vestiges à Panthera gombaszoegensis et suivant la palynologie des niveaux fossilifères (Argant 1980), retient que cette espèce se serait développée surtout dans un biotope forestier. Toutefois comme Panthera gombaszoegensis était aussi représentée lors des épisodes froids du Pléistocène moyen, A. Argant (1989) la considère comme relativement ubiquiste.

\section{Grotte de L'Escale (Bouches-Du-Rhône)}

6 Ce gisement a livré des vestiges dont l'âge se place entre 0,4 et $1 \mathrm{Ma}$. Le matériel se compose essentiellement de fragments de crâne et de dents de quatre jeunes adultes (Bonifay 1971). Cet auteur a constaté que ce grand félin présentait à la fois des caractères de Guépard (Acinonyx), de Lion et de Megantereon mais aussi des caractères qui lui sont propres. Les caractères originaux de ce fossile sont essentiellement liés à la capacité de broyer les os d'après M.-F. Bonifay (1971), ce qui est rare pour un félin. Proche de celui décrit à Gombaszög mais présentant des caractères particuliers, ce félin a été distingué par M.-F. Bonifay (op. cit.) des autres félins au niveau du genre, sous le nom de Jansofelis vaufreyi. Toutefois pour H. Hemmer (1972), Jansofelis vaufreyi serait un synonyme de Panthera gombaszoegensis, les restes présents à l'Escale se rapportant à des individus en cours de croissance. Quant à Rimmer (in Hemmer 1972), il estime que les particularités de l'individu trouvé dans le Sud de la France ne justifient une distinction qu'au niveau sous-spécifique.

7 Il faut cependant envisager de façon tout à fait probable une évolution de Panthera gombaszoegensis au cours du Pléistocène. 


\section{Artenac (Charente)}

8 Ce site a livré de nombreux restes de carnivores tels que ceux rapportés aux genres Ursus, Canis ou à l'espèce Dinobastis latidens, ... ainsi que des restes d'un félin que J.-F. Tournepiche (1996) a décrit comme appartenant à Panthera gombaszoegensis. Ces vestiges proviennent des niveaux inférieurs de la grotte datés entre le Villafranchien récent et la fin du Mindel.

9 Les fouilles ont notamment mis au jour deux crânes rapportables à ce taxon, plus ou moins complets, datant du Mindel.

10 Récemment, un quatrième site, la Grotte XIV en Dordogne, a livré des vestiges qui appartiendraient à l'espèce Panthera gombaszoegensis. Nous avons étudié les restes mis au jour dans ce site et nos résultats sont exposés dans les paragraphes suivants.

\section{La grotte XIV}

11 Cette grotte se situe en Dordogne, sur la commune de Cénac et Saint-Julien. La séquence chronologique des dépôts de ce site s'étale de 500 à 600000 ans pour l'ensemble inférieur, à 120000 ans pour l'ensemble supérieur (Guadelli 1998 ).

12 Le matériel étudié qui provient des ensembles inférieur et moyen, se compose de deux mandibules (B1-384, mandibule droite complète; B1-238, branche montante cassée d'une mandibule gauche) et de restes d'os post-crâniens.

\section{Mandibules}

Les deux mandibules sont de même taille (fig. 1 et $2 ;$ tabl. 1). La branche montante du spécimen B1-384 est moins haute que chez Panthera spelaea et son départ est assez abrupt. La fosse massétèrienne est assez vaste et s'arrête nettement avant la racine distale de la première molaire inférieure (M/1). A. Argant (1989) signale que $H$. Hemmer et G. Schütt (1969) et M.-F. Bonifay (1971) ont constaté les mêmes caractères sur les fossiles qu'ils ont découverts. Les bords supérieur et inférieur de l'os mandibulaire sont chaque fois à peu près rectilignes et parallèles sur toute leur longueur. A la partie antérieure de la symphyse, on remarque une proéminence qui n'est visible ni sur les fossiles de l'Escale (Bonifay 1971) ni sur ceux de Château (Argant 1989). On note également que la hauteur de la branche horizontale de la mandibule paraît importante.

14 En vue occlusale, nous pouvons observer que la série des dents jugales $(\mathrm{P} / 3, \mathrm{P} / 4, \mathrm{M} / 1)$ forme une convexité vers la face vestibulaire. On s'aperçoit que sur la mandibule B1-384, la M/1 a un protoconide plus élevé que le paraconide, observation impossible à effectuer sur l'autre mandibule compte tenu de l'usure de la dent (tabl. 2). Il n'y a pas d'espace entre les dents jugales ce qu'a noté M.-F. Bonifay (op. cit.) pour le fossile de l'Escale. La $\mathrm{P} / 3$ est petite par rapport à la $\mathrm{P} / 4$ particulièrement puissante. Le protoconide de cette dernière est pointu et presque aussi haut que celui de la $\mathrm{M} / 1$, il est encadré de deux petits tubercules : un antérieur et l'autre postérieur. La série dentaire étant en " tuile de toit", la partie distale de $\mathrm{P} / 4$ est, du côté vestibulaire, plus en arrière que le bord mésial de la M/1. 
La $\mathrm{M} / 1$ est à peine plus grosse que la $\mathrm{P} / 4$ : ce que nous pouvons constater figure 2 et dans le tableau 2. Il apparaît figure 3 que les $\mathrm{M} / 1$ découvertes à Artenac sont de même taille que celles provenant de la Grotte XIV, ce qui est un argument de plus à l'attribution des fossiles de cette dernière grotte à Panthera gombaszoegensis.

Fig. 1 - Panthera gombaszoegensis, mandibule gauche, vue latérale. GR XIV, B1-238 c. 15, Grotte XIV

Fig. 1 - Panthera gombaszoegensis, left mandibula, lateral view. GR XIV, B1-238 c. 15, Grotte XIV

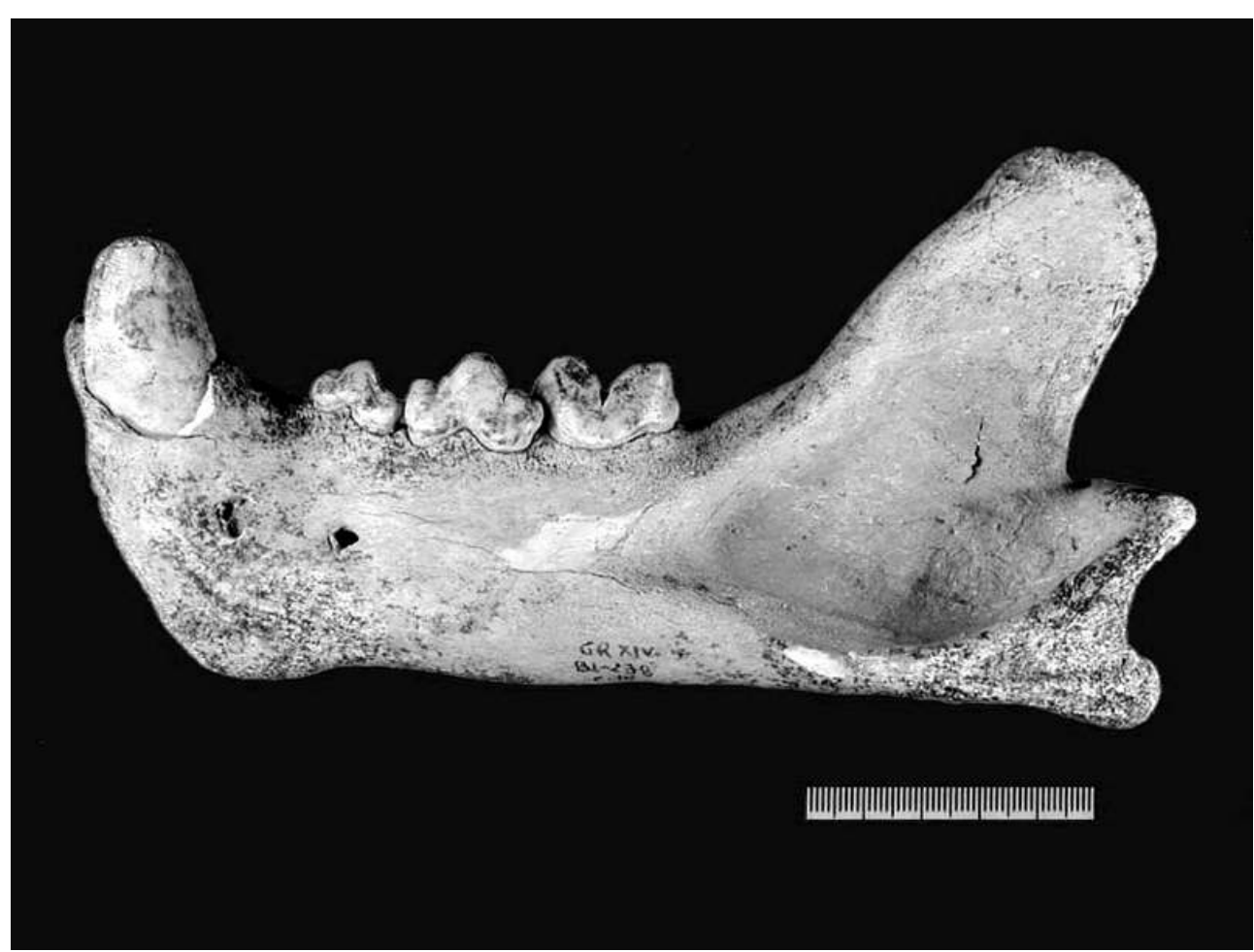


Fig. 2 - Panthera gombaszoegensis, mandibule droite, vue latérale. GR XIV, B1-384 c. 15, Grotte XIV Fig. 2 - Panthera gombaszoegensis, right mandibula, lateral view. GR XIV, B1-384 c. 15, Grotte XIV

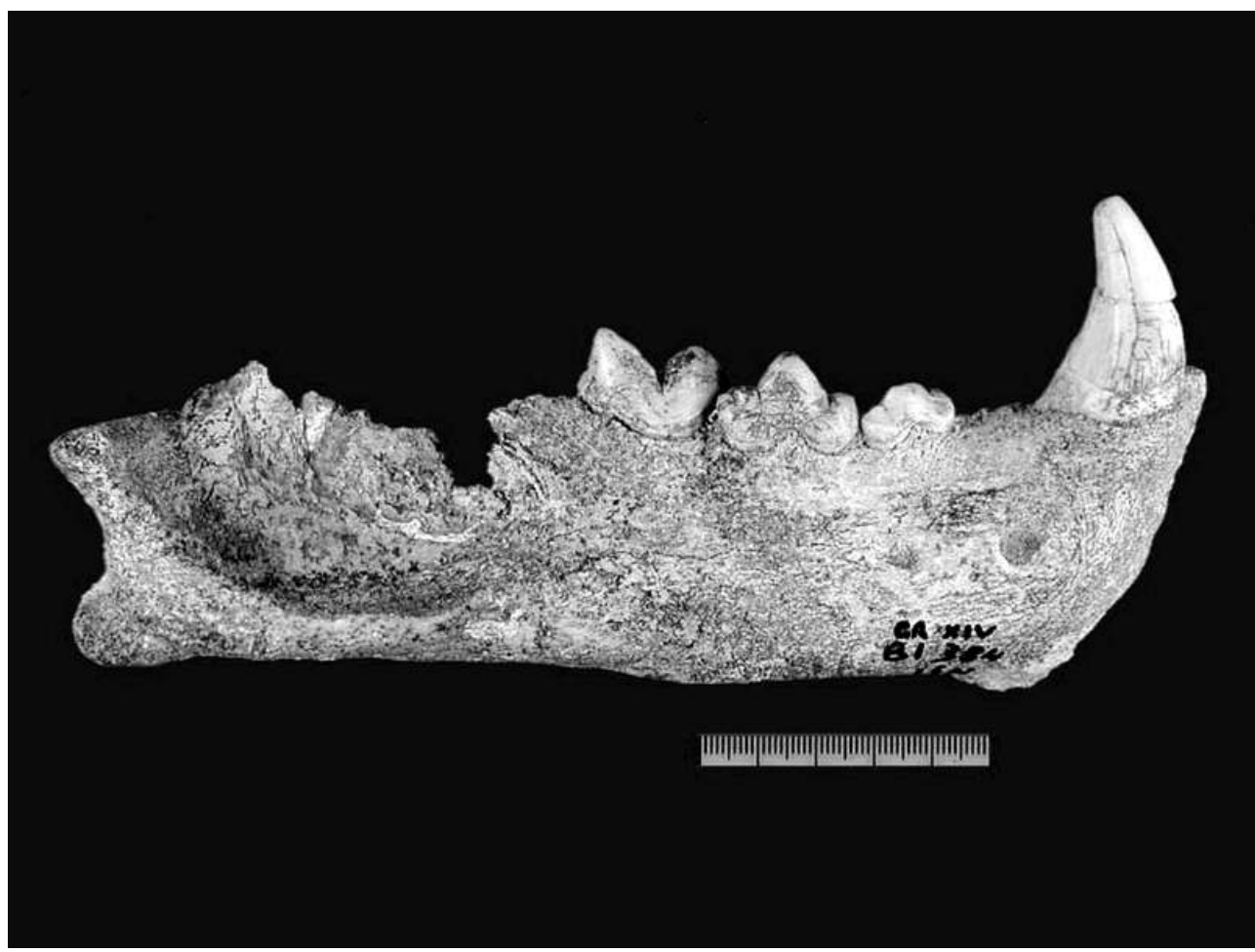

Tabl. 1 - Panthera gombaszoegensis : mandibule. 1 : sous la M1 - 2 : en avant de la P4 Table 1 - Panthera gombaszoegensis : mandibula. 1 : under the $M 1$ - 2 : forward the P4

\begin{tabular}{|c|c|c|c|c|c|c|c|c|}
\hline & \multicolumn{2}{|c|}{ Grotte XIV } & $\begin{array}{c}\text { Château } \\
\text { Argant } \\
1989\end{array}$ & \multirow{2}{*}{ 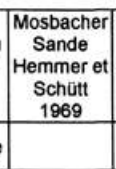 } & \multirow{2}{*}{\begin{tabular}{|c|}
$\begin{array}{c}\text { Val d'Amo } \\
\text { Del } \\
\text { Campana } \\
1915\end{array}$ \\
2 individus \\
\end{tabular}} & \multirow{2}{*}{$\begin{array}{c}\begin{array}{c}\text { Olivola } \\
\text { Del } \\
\text { Campana } \\
1915\end{array} \\
\end{array}$} & \multirow{2}{*}{$\begin{array}{l}\text { L'Escale } \\
\begin{array}{l}\text { M.F. Bonifay } \\
\text { Jansofelis vaufreyi }\end{array} \\
\begin{array}{l}\text { Moyenne (nbre individus } \\
\text { valeur mini - valeur maxi) }\end{array}\end{array}$} \\
\hline & & $\begin{array}{c}\text { B1-384 } \\
\text { c15 }\end{array}$ & $\begin{array}{c}\text { B1-238 } \\
c 15\end{array}$ & moulage & & & & \\
\hline & $\begin{array}{l}\text { Longueur entre le bord mésial de l'alvéole de la } \\
\text { I1 et le processus condylaire }\end{array}$ & 194,00 & 196,00 & & & & & \\
\hline & $\begin{array}{l}\text { Longueur entre le bord mésial de l'alvéole de la } \\
\text { l1 et le processus angulaire }\end{array}$ & 190,00 & 192,00 & & & & & \\
\hline & $\begin{array}{l}\text { Longueur entre le bord mésial de l'alvéole de la } \\
\text { 11 et le processus coronoide }\end{array}$ & ? & 198,00 & & & & & \\
\hline & Hauteur processus angulaire - coronion & $?$ & 97,00 & & & & & \\
\hline & Longueur $\mathrm{P} / 3-\mathrm{M} / 1$ ou alvéoles & 65,30 & 64,14 & 66,40 & 62,00 & & $?$ & $62,7(4 / 61,8-64,0)$ \\
\hline 6 & Longueur diastème C-P/3 & 16,66 & 17,16 & 18,00 & 16,00 & $17,0 \quad 22,0$ & 20 & $6,57(4 / 3-9,1)$ \\
\hline & Hauteur branche mandibulaire derrière $M / 1$ & 47,52 & 45,26 & 44,50 & $?$ & $?$ & $?$ & $29,73(6 / 26,2-33,0)^{\prime}$ \\
\hline 8 & Hauteur branche mandibulaire devant $\mathrm{P} / 3$ & 47,38 & 45,12 & 44,00 & 38,50 & $?$ & $?$ & \\
\hline 9 & Hauteur branche mandibulaire au niveau de $\mathrm{P} / 3$ & 46,56 & 46,10 & & & 37.036 .0 & 28,5 & $31,63(6 / 30,2-32,0)^{2}$ \\
\hline 10 & Longueur de l'alvéole de $\mathrm{P} / 3$ & 18,14 & 16,88 & & & $\begin{array}{|ll|}16,5 & 16,2 \\
\end{array}$ & 14 & $17,07(4 / 16,8-17,5)$ \\
\hline & Longueur de l'alvéole de P/4 & 24,42 & 24,00 & & & & & $23,43(6 / 22,6-24,5)$ \\
\hline & Longueur de l'alvéole de M/1 & 26,10 & 24,66 & & 24,20 & $22,6 \quad 23,3$ & & $25,37(6 / 24,8 \cdot 26,3)$ \\
\hline & Diamètre transverse au niveau de C & 25,72 & 25,24 & 21,50 & & & & \\
\hline & Diamètre transverse en avant de $\mathrm{P} / 3$ & 22,26 & 21,04 & & & & & \\
\hline & Diamètre transverse en arrière de M/1 & $?$ & 24,80 & 26,20 & & & & \\
\hline & Diamètre transverse du condyle d'articulation & 45,82 & 42,44 & & & & & \\
\hline
\end{tabular}

\section{Squelette post-cranien}

\section{$2^{\text {ème }}$ Vertèbre cervicale (axis)}

16 L'axis découvert à la Grotte XIV est en bon état de conservation et apparaît robuste (fig. $4 a, 4 b)$, toutefois il est difficile de le comparer aux deuxièmes vertèbres cervicales 
d'autres espèces de Panthera car très peu ont été retrouvées dans un état permettant leur étude.

Dans sa partie crâniale, cette vertèbre est plus large que celle d'un jeune lion conservé à l'I.P.G.Q. de l'Université de Bordeaux I (61,8 $\mathrm{mm} ; 65,7 \mathrm{~mm}$ pour l'axis de la Grotte XIV). Le processus épineux, très long et très oblique, dépasse très nettement vers l'arrière le corps de la vertèbre (fig. 4a). Ce processus est plus oblique vers le haut et vers l'arrière que chez un jeune lion actuel. En revanche, les articulations de cette vertèbre avec l'atlas de ce jeune félin sont identiques à celles du félin de la Grotte XIV (fig. 4b). Les processus articulaires caudaux sont détachés du processus épineux contrairement à ce que l'on peut voir chez les lions actuels.

Tabl. 2 - Panthera gombaszoegensis, dents inférieures. Grotte XIV Table 2 - Panthera gombaszoegensis, lower teeth. Grotte XIV

\begin{tabular}{|c|c|c|c|c|c|c|c|c|}
\hline \multirow{2}{*}{ Dents } & Référence & Longueur & Largeur & $\begin{array}{c}\text { Hauteur de la } \\
\text { couronne } \\
(\mathrm{HC})\end{array}$ & $\begin{array}{c}\mathrm{H.C} \\
\text { protoconide }\end{array}$ & $\begin{array}{c}\mathrm{HC} \\
\text { paraconide }\end{array}$ & $\begin{array}{c}\text { MD } \\
\text { protoconide }\end{array}$ & $\begin{array}{r}\text { MD } \\
\text { paraconide }\end{array}$ \\
\hline \multirow{2}{*}{$\mathrm{C}$} & $\mathrm{B} 1-384$ & 21,22 & 14,66 & 33,88 & & & & \\
\hline & $\mathrm{B} 1-238$ & 19,64 & 15,10 & 16,54 & & & & \\
\hline \multirow{2}{*}{$\mathrm{P} / 3$} & $\mathrm{~B} 1-384$ & 16,74 & 7,20 & 7,90 & & & & \\
\hline & $\mathrm{B} 1-238$ & 17,20 & 8,18 & 8,82 & & & & \\
\hline \multirow{2}{*}{$\mathrm{P} / 4$} & $\mathrm{~B} 1-384$ & 23,74 & 10,54 & 13,02 & & & & \\
\hline & $\mathrm{B} 1-238$ & 24,00 & 12,10 & 12,72 & & & & \\
\hline \multirow{2}{*}{$\mathrm{M} / 1$} & $\mathrm{~B} 1-384$ & 26,14 & 12,32 & & 13,44 & 10,22 & 13,24 & 12,66 \\
\hline & $\mathrm{B} 1-238$ & 25,62 & 12,54 & & 11,38 & 10,44 & 11,94 & 11,00 \\
\hline
\end{tabular}

Fig. 3 - Panthera, première molaire inférieure, diamètre mésio-distal (DMD) en fonction du diamètre vestibulo-lingual (DVL). Artenac (Tournepiche 1993) et Grotte XIV

Fig. 3 - Panthera, first lower molar, mésio-distal diameter (DMD) as function of vestibulo-lingual diameter (DVL). Artenac (Tournepiche 1993) et Grotte XIV

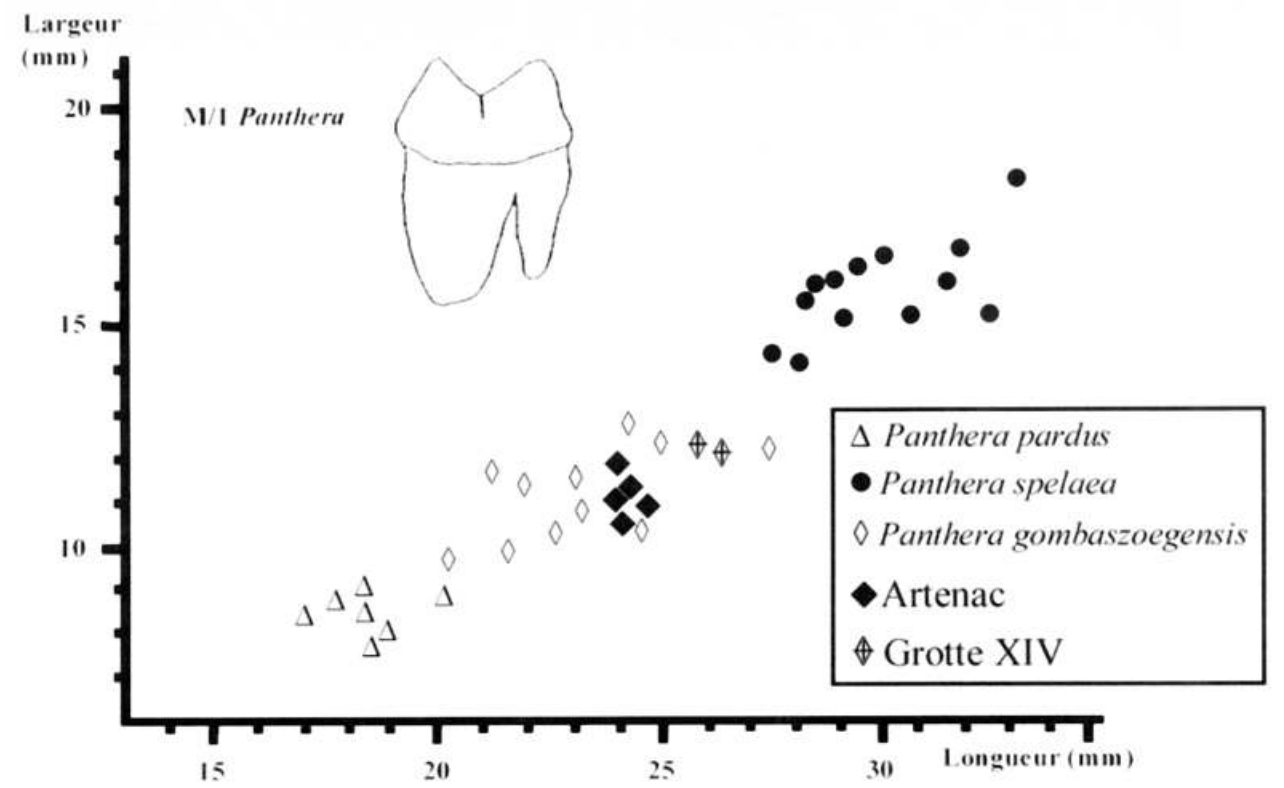


Fig.4 a - Panthera gombaszoegensis, axis, vue latérale. Grotte XIV Fig. 4 a - Panthera gombaszoegensis, axis, lateral view. Grotte XIV

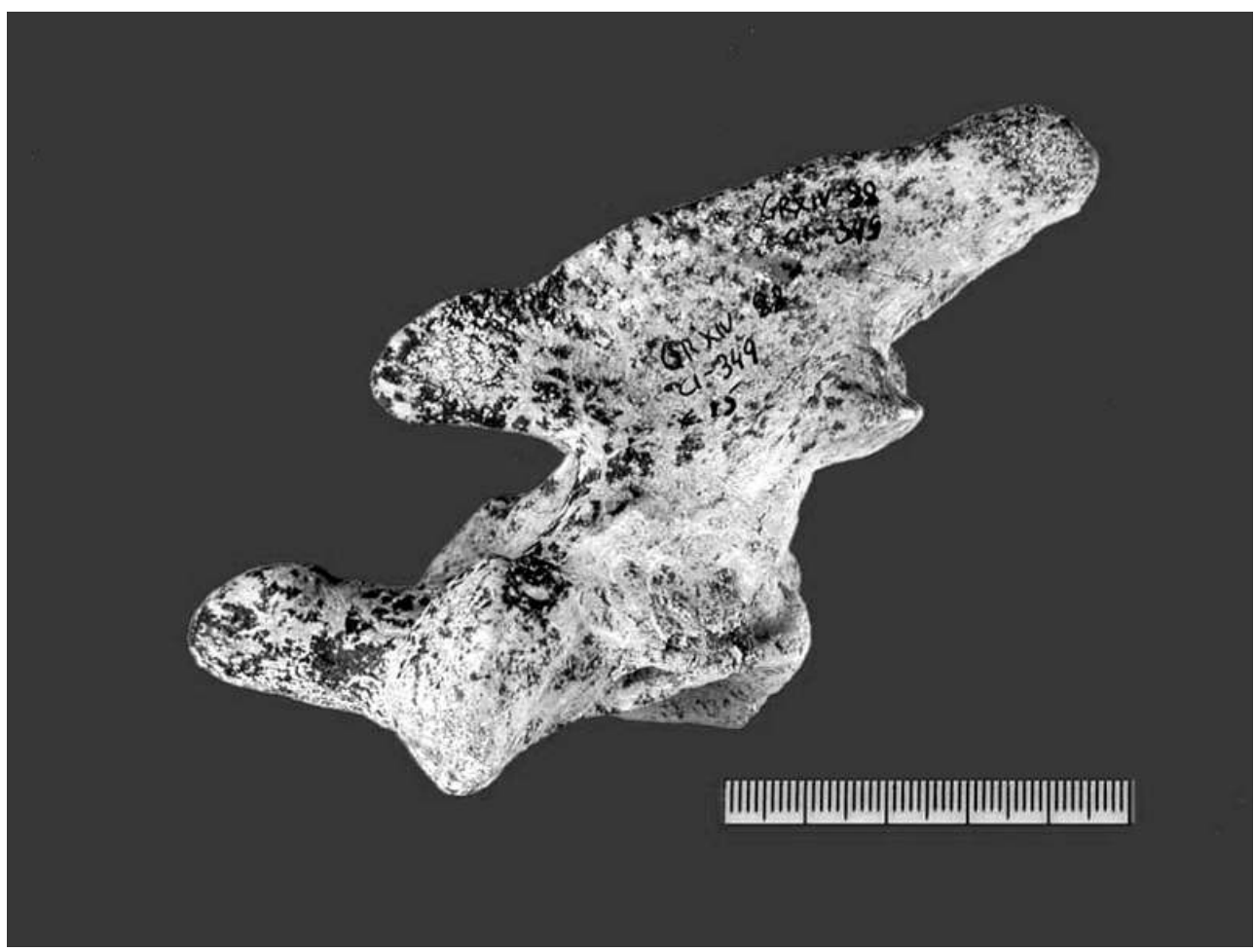

Fig. 4b - Panthera gombaszoegensis, axis, vue crâniale. Grotte XIV Fig.4b - Panthera gombaszoegensis, axis, cranial view. Grotte XIV

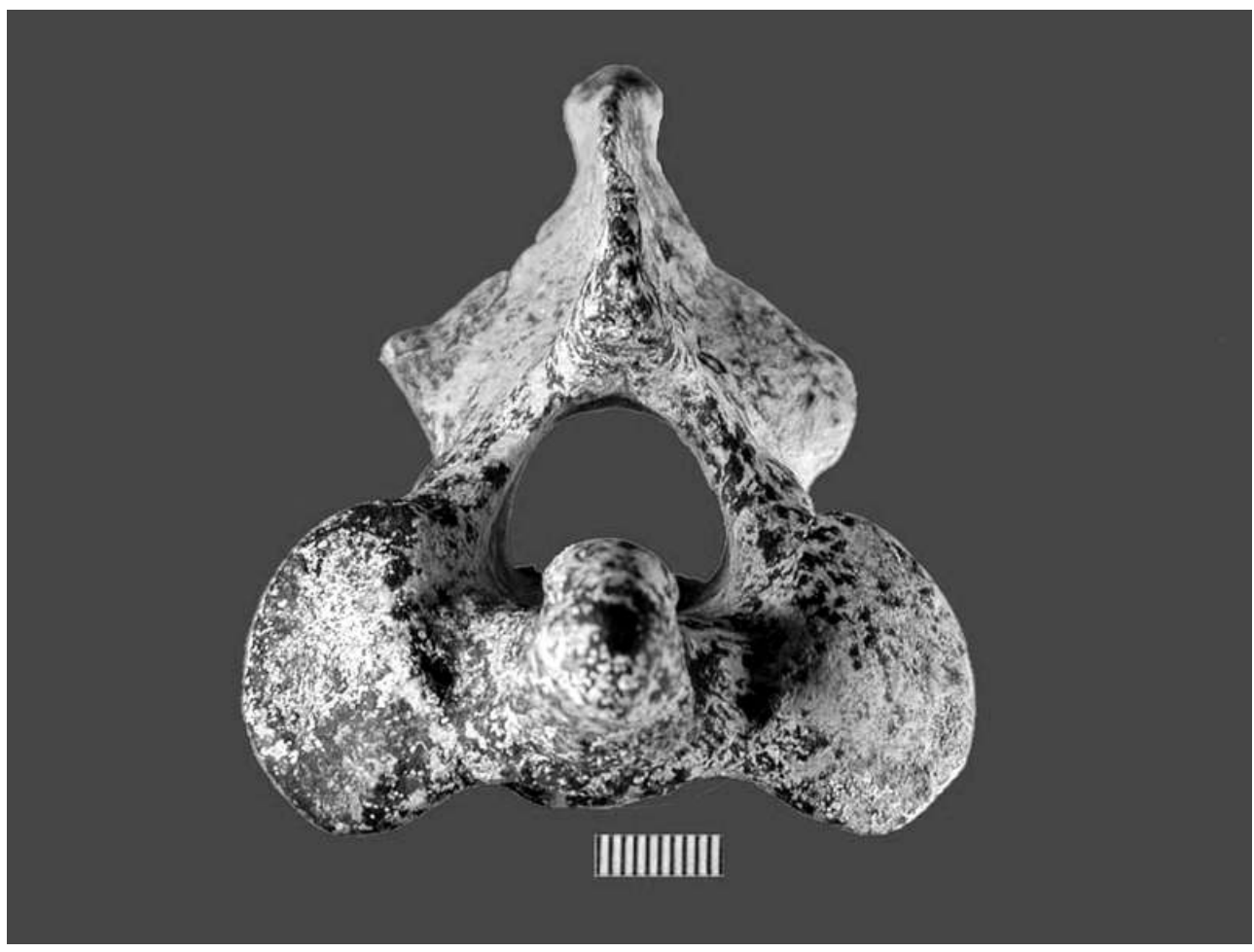




\section{Radius}

Nous avons comparé cet os à celui du Lion actuel car nous n'avons pas pu nous référer à des ossements fossiles. Ce radius, dont on n'a que l'extrémité distale (fig. 5), possède une diaphyse plus aplatie antéro-postérieurement que chez le Lion actuel. Le processus styloïde radial est présent mais très peu développé. L'articulation avec le carpe est très rectangulaire et paraît creusée en son milieu. La largeur de la diaphyse est comparable à celle du Lion actuel. Sur la face dorsale de cette diaphyse, la proéminence au-dessus du processus styloïde est plus développée et plus haute. La face palmaire du radius du félin de la Grotte XIV était endommagée donc il n'a pas été possible d'observer et de juger du développement des insertions musculaires.

Fig. 5 - Panthera gombaszoegensis, radius droit. Grotte XIV Fig. 5 - Panthera gombaszoegensis, right radius. Grotte XIV

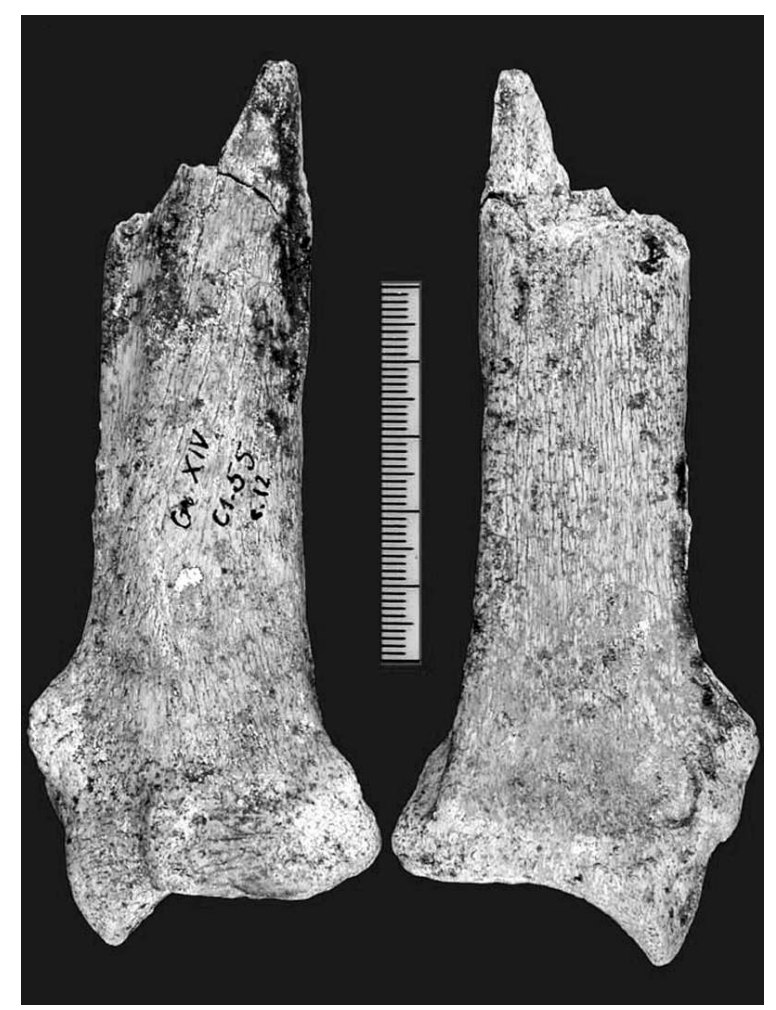

\section{Métacarpien V}

Sa partie proximale est plus globuleuse que sa partie distale. Si on le compare à un métacarpien V de Panthera spelaea de Malarnaud (Dufour 1989), il est beaucoup plus petit et plus court. En comparant les mesures des 5èmes métacarpiens des différents carnivores (tabl. 3), on s'aperçoit que celui du félin de la Grotte XIV est plus proche en taille de ceux de Panthera gombaszoegensis de Château (Argant 1989) que de ceux de $P$. pardus et $P$. mosbachensis. Ce métacarpien se situe entre celui plus robuste d'un lion actuel et celui plus fin et plus court d'une panthère actuelle. Mais il possède la même morphologie que celui de Panthera pardus. Par rapport au Lion actuel, la facette articulaire en relation avec le 4ème métacarpien est plus droite et dans l'axe de l'os. C'est ce que l'on retrouve également chez la Panthère actuelle. 
Tabl. 3 - Panthera gombaszoegensis, Métacarpien V. Grotte XIV et Château (Argant, 1989) Table 3 - Panthera gombaszoegensis, Métacarpal V. Grotte XIV and Château (Argant, 1989)

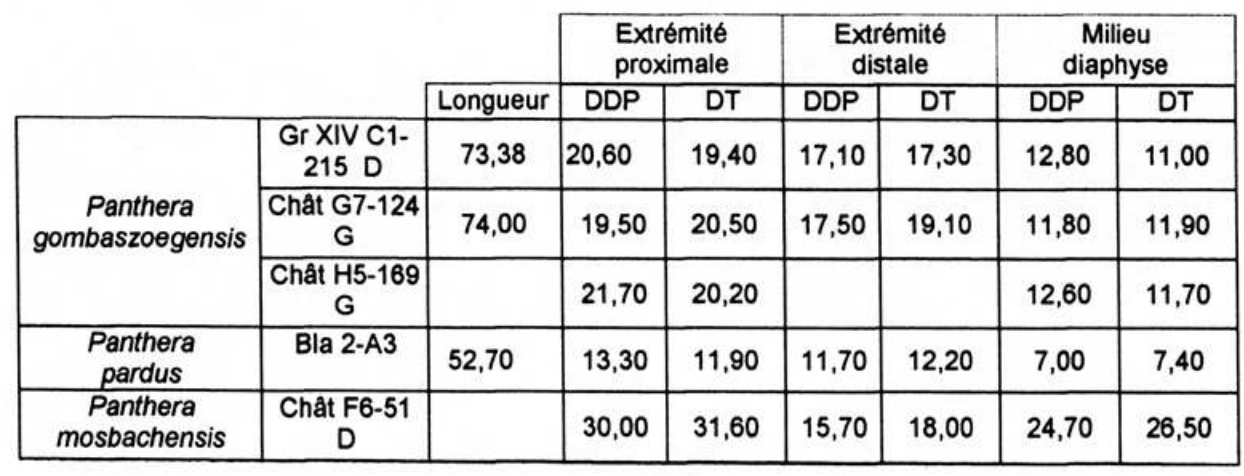

DDP : diamètre dorso-plantaire, DT : diamètre transversal

\section{Fémur}

La diaphyse de ce fémur a une section arrondie sur la face dorsale et plane sur la face plantaire. Le grand trochanter, cassé, ne paraît pas avoir été très volumineux. La trochlée fémorale est beaucoup moins développée et moins épaisse que celle d'un lion actuel. Par ailleurs, relativement au fémur du Lion, celui découvert à la Grotte XIV possède une diaphyse plus robuste. Cette dernière semble plus torse ce qui est observable aussi sur les fémurs des panthères. La face plantaire de cet os, dans sa moitié distale, possède au milieu de la diaphyse une légère carène, c'est pourquoi cette face paraitt moins plate que celle du Lion actuel. La tête articulaire du fémur n'a aucun caractère particulier, elle est comparable à celle du Lion mais son col paraît plus court.

\section{Tibia et Talus}

21 Le tibia est malheureusement cassé, il ne nous reste que la moitié distale. Il paraît assez robuste par rapport à celui du Lion actuel. Les deux faces articulaires sont plus ou moins obliques, peu profondes mais la plus latérale forme une gouttière. Cette articulation distale paraît plus aplatie antéro-postérieurement chez le félin de la Grotte XIV que chez le Lion actuel. Les diamètres transversal et antéro-postérieur paraissent plus importants pour le tibia trouvé à la Grotte XIV que pour celui du Lion actuel.

Le talus (fig. 6) est très bien conservé et ses dimensions (tabl. 4) comparées à celles des deux talus trouvés à Château en Bourgogne (Argant 1989) semblent assez proches.

Tabl. 4 - Panthera gombaszoegensis, Talus. Grotte XIV et Château (Argant, 1989)

Table 4 - Panthera gombaszoegensis, Talus. Grotte XIV and Château (Argant, 1989)

\begin{tabular}{|l|c|c|c|c|c|c|c|c|}
\cline { 4 - 8 } \multicolumn{1}{c|}{} & \multicolumn{4}{c|}{} & \multicolumn{2}{c|}{$\begin{array}{c}\text { Surface } \\
\text { articulaire }\end{array}$} & \multicolumn{1}{c|}{} \\
\cline { 2 - 8 } \multicolumn{1}{c|}{} & DDP & $\begin{array}{c}\text { DT } \\
\text { maximum }\end{array}$ & $\begin{array}{c}\text { DT } \\
\text { trochlée }\end{array}$ & DDP & DT & $\begin{array}{c}\text { DT } \\
\text { col }\end{array}$ & $\begin{array}{c}\text { DDP } \\
\text { col }\end{array}$ & $\begin{array}{c}\text { DDP } \\
\text { maximum }\end{array}$ \\
\hline Gr XIV S 96-146 & 49,62 & & 30,00 & 22,38 & 28,38 & 23,90 & 22,90 & $\sim 35,00$ \\
\hline Chât F.7-7 & 51,20 & & & 26,50 & 27,30 & 22,40 & 20,80 & 32,50 \\
\hline Chât G.6-121 & 50,00 & & & 26,20 & 27,30 & 22,60 & 21,30 & $\sim 31,5$ \\
\hline
\end{tabular}

DDP : diamètre dorso-plantaire, DT : diamètre transversal 
Fig. 6 - Panthera gombaszoegensis, astragale droit. Grotte XIV Fig. 6 - Panthera gombaszoegensis, right astragalus. Grotte XIV

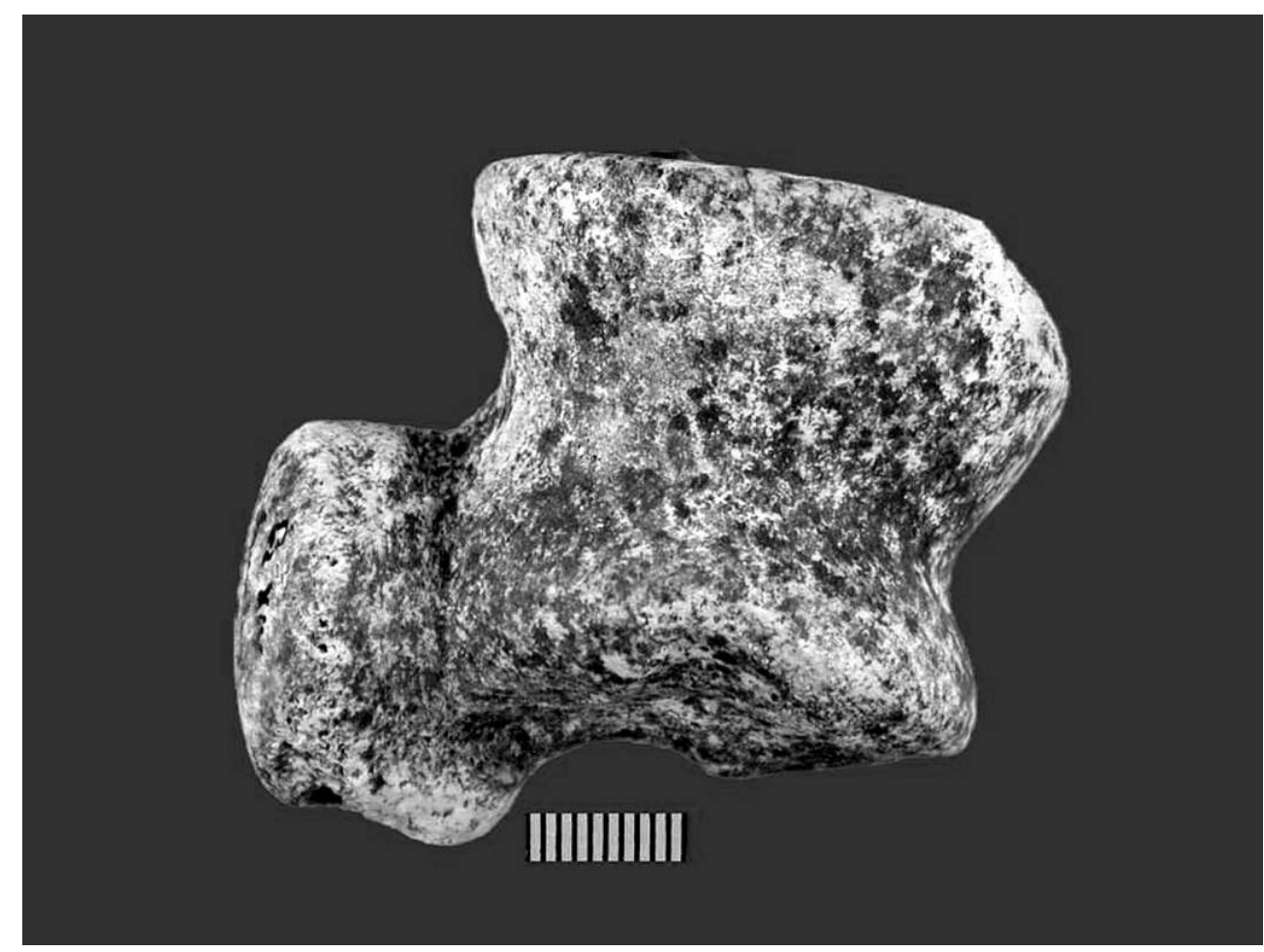

Par rapport au Lion actuel, ce talus est moins haut (Gr. XIV : $49 \mathrm{~mm}$; Lion actuel : 53,14 $\mathrm{mm}$ ), paraît plus comprimé dans le sens dorso-plantaire et sa trochlée est un peu plus large avec une gorge un peu moins profonde. L'articulation avec le naviculaire apparaît plus trapue et son col plus court. Au niveau de cette articulation, en position médiale, on peut observer un petit tubercule qui n'existe pas chez les autres félins. Sur sa face plantaire, la plus petite facette articulaire pour le calcanéum est moins vaste et plus ronde que chez le Lion actuel.

Le tibia et le talus semblent appartenir au même individu.

\section{Phalanges (fig. 7)}


Fig. 7a - Panthera gombaszoegensis, phalange I, face supérieure. Grotte XIV. 7b : Panthera gombaszoegensis, phalange II, face supérieure. Grotte XIV. 7c : Panthera gombaszoegensis, phalange II, face inférieure. Grotte XIV

Fig. 7a - Panthera gombaszoegensis, phalanx I, upper side. Grotte XIV. $7 \mathrm{~b}$ : Panthera gombaszoegensis, phalanx II, upper side. Grotte XIV. 7c : Panthera gombaszoegensis, phalanx II, lower side. Grotte XIV
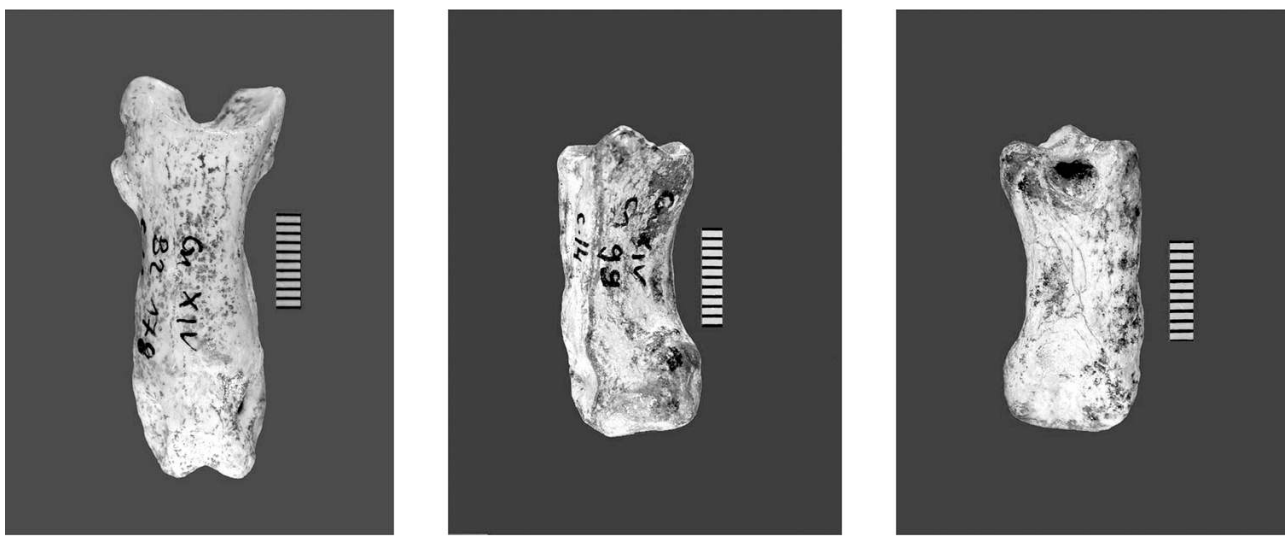

\section{Phalange proximale (phalange I)}

La phalange appartenant au fossile découvert à la Grotte XIV paraît un peu plus petite que celles trouvées par A. Argant (1989) à Château (tabl. 5). Elle est très arquée et son articulation proximale comprend une échancrure assez vaste. Cette phalange est un peu plus large et longue que celle d'une panthère actuelle mais beaucoup moins robuste que celle d'un jeune lion. Néanmoins, sur cet os, en vue dorsale, on peut repérer, dans sa partie proximale, un petit bourrelet proéminent du côté gauche de la diaphyse et un creux du côté droit (fig. 7a).

Tabl. 5 - Panthera gombaszoegensis, Phalanges. Grotte XIV et Château (Argant, 1989) Table 5 - Panthera gombaszoegensis, Phalanx. Grotte XIV and Château (Argant, 1989)

\begin{tabular}{|c|c|c|c|c|c|c|c|c|}
\hline & & \multicolumn{2}{|c|}{$\begin{array}{l}\text { Extrémité } \\
\text { proximale }\end{array}$} & \multicolumn{2}{|c|}{$\begin{array}{c}\text { Extrémité } \\
\text { distale }\end{array}$} & \multicolumn{2}{|c|}{$\begin{array}{c}\text { Milieu } \\
\text { diaphyse }\end{array}$} \\
\hline & & Longueur & DDP & DT & DDP & DT & DDP & DT \\
\hline \multirow{3}{*}{ Phalange I } & $\begin{array}{l}\text { Gr XIV B2- } \\
178\end{array}$ & 42,30 & 13,30 & 17,40 & 10,70 & 14,10 & 11,70 & 12,80 \\
\hline & Chât F7-2 & 50,80 & 17,50 & 21,60 & 12,60 & 16,50 & 11,90 & 17,30 \\
\hline & Chât F7-11 & 48,50 & 14,80 & 19,60 & 11,60 & 15,40 & 10,60 & 13,70 \\
\hline \multirow{4}{*}{ Phalange II } & $\begin{array}{l}\text { Gr XIV C1- } \\
99 \mathrm{G} \\
\end{array}$ & 32,10 & 13,90 & 15,00 & 9,12 & 14,30 & 9,60 & 11,90 \\
\hline & $\begin{array}{l}\text { Gr XIV 96- } \\
148 \mathrm{G} \\
\end{array}$ & 38,14 & $\sim 15,2$ & & 10,70 & 15,30 & 9,80 & 10,90 \\
\hline & $\begin{array}{l}\text { Chât G7- } \\
93 \text { G }\end{array}$ & 34,50 & 15,30 & 16,50 & 11,80 & 15,40 & 10,10 & 11,00 \\
\hline & $\begin{array}{l}\text { Chât G7- } \\
91 \text { D }\end{array}$ & 34,40 & 15,20 & 16,20 & 11,60 & 15,80 & 9,90 & 12,00 \\
\hline
\end{tabular}

DDP : diamètre dorso-plantaire, DT : diamètre transversal

\section{Phalanges intermédiaires (phalanges II) :}

Les deux 2èmes phalanges trouvées à la Grotte XIV sont, elles aussi, arquées et comprimées transversalement (fig. $7 \mathrm{~b}$ ). Comme chez tous les félins, elles possèdent une dépression ronde dans la partie proximale de la face plantaire (fig. 7c). La taille de ces 
phalanges est plus proche de celle des phalanges d'un jeune lion que de celle des phalanges d'une panthère actuelle.

\section{Phalange unguéale (phalange III)}

Du fait de sa taille, cette phalange paraît avoir porté une griffe assez imposante.

\section{Conclusion}

28 A partir des mesures et des descriptions des différents restes découverts à la Grotte XIV, nous pouvons remarquer que ce félin possède plusieurs caractères montrant son appartenance à l'espèce Panthera gombaszoegensis :

- la branche horizontale des mandibules est haute et ses bords supérieur et inférieur sont rectilignes et parallèles ; la vaste fosse massétérienne s'arrête avant la racine distale de la $\mathrm{M} / 1$,

- les carnassières sont robustes,

- le talus est comprimé et son col est court. Sur sa face plantaire, la petite facette articulaire calcanéenne médiale est petite et ronde.

Cependant, Panthera gombaszoegensis découvert à la Grotte XIV présente des caractères qui n'ont pas été évoqués dans la littérature à notre disposition; ils sont peut-être propres à la forme de ce site ou caractéristique de l'espèce sans que nous ne puissions trancher pour l'instant pour l'une ou l'autre des deux hypothèses :

- le processus épineux de l'axis est long et oblique,

- les mandibules possèdent dans la partie inférieure de la symphyse une proéminence,

- la diaphyse du fémur est torse, elle possède une carène dans sa moitié distale, sur la face postérieure et son col est assez court,

- la face palmaire du troisième cunéiforme est volumineuse et son articulation avec le métacarpien III est vaste,

- la phalange proximale possède, à la partie proximo-médiale, un petit tubercule.

30 A ces quelques détails près, ce félin ressemble beaucoup à ceux de Château (Argant 1989). Les particularités notées ci-dessus relativement aux félins de Château ou à celui de l'Escale (Bonifay 1971) relèvent peut-être d'une adaptation régionale.

Panthera gombaszoegensis présent à la Grotte XIV vivait en compagnie d'autres carnivores: Ursus deningeri, Dinobastis latidens, Panthera (leo) spelaea, Lynx sp., Canis etruscus, Vulpes praeglacialis, sous un climat frais et humide dans un environnement forestier de type chênaie (Guadelli 1998). Ceci est comparable aux résultats d'A. Argant (1989) qui, à partir des données palynologiques de Château (Argant 1980) associe Panthera gombaszoegensis à un milieu forestier, tempéré frais. On observe les mêmes conditions climato-écologiques à l'Escale (Bonifay 1971).

32 Les fouilles de la Grotte XIV ont permis de mettre au jour des restes de Panthera spelaea et de Panthera gombaszoegensis dans les mêmes couches de l'ensemble moyen et de l'ensemble inférieur (Guadelli 1998) ce qui indiquerait, semble-t-il, que ces deux espèces n'entraient pas en compétition. Selon A. Argant (1989), Panthera gombaszoegensis n'était pas non plus directement en concurrence avec Panthera mosbachensis.

Nous avons voulu, dans cette étude, apporter quelques éléments à la connaissance de l'espèce Panthera gombaszoegensis mais il reste des zones d'ombre au niveau 
taxonomique, Jansofelis vaufreyi doit-il être mis en synonymie avec Panthera gombaszoegensis malgré ses caractères propres?

Le Félin de Gombaszög était une espèce qui a couvert une grande étendue chronologique et géographique (Villafranchien supérieur; Italie : Val d'Arno, Olivola ; Pays Bas : Tegelen. Pléistocène moyen; Hongrie : Gombaszög; Allemagne : Mosbach ; France: l'Escale, Château, Artenac, la Grotte XIV). Il peut donc être représenté par plusieurs formes, différentes entre elles au niveau sous spécifique, qui auraient eu une évolution propre au sein d'aires géographiques non superposées.

\section{BIBLIOGRAPHIE}

ARGANT A. 1989 - Carnivores quaternaires de Bourgogne, Thèse de doctorat : Université de Lyon I, $\mathrm{n}^{\circ}$ 152-89, 321 p., $9 \mathrm{pl}$.

ARGANT J. 1980 - Analyse palynologique de trois gisements du Quaternaire de Saône-et-Loire : Château, Solutré, Chassey, D.E.S. Sci. Nat. Sci. de la Terre : Université de Lyon I, 76 p., 18 fig., 10 tabl.

BONIFAY M.-F. 1971- Carnivores quaternaires du Sud - Est de la France. Mémoires du Muséum national d'Histoire Naturelle, Paris, Ed. du Muséum, t. XXII, 377p., 76 fig., 109 tabl., 27 pl.

DUFOUR R. 1989 - Les carnivores pléistocènes de la caverne de Malarnaud (Ariège), (Collection E. Harle, Muséum d'Histoire Naturelle de Bordeaux), DES de Sciences naturelles : Université Bordeaux I, 455 p., ill.

GUADELLI J.-L. 1998 - Grotte XIV, Cénac et Saint-Julien, Bilan scientifique de la Région Aquitaine, Direction Régionale des Affaires Culturelles, SRA, Bordeaux.

GUERIN C. et PATOU-MATHIS M. 1996 - Les grands mammifères plio-pléistocènes d'Europe. Paris, Masson, 291 p., ill.

HEMMER H. 1972 - Zur systematischen Stellung von “Jansofelis vaufreyi” Bonifay 1971 und von "Felis lunellensis" Bonifay 1971, aus dem Pleistozän Südfrankreichs (Carnivora, Felidaea). N.Jb.palaont, Mh., Stuttgart, p. 215-223, 2 fig., 2 tabl.

HEMMER H., KAHLKE R.-D., VEKUA A. K. 2001 - The Jaguar - Panthera onca gombaszoegensis (Kretzoi, 1938) (Carnivora : Felidae) in the late lower Pleistocene of Akhalkalaki (South Georgia ; Transcaucasia) and its evolutionary and ecological significance. Géobios, 34, 4, p. 475-486.

HEMMER H. et SCHÜTT G. 1969 - Ein Unterkiefer von Panthera gombaszöegensis (Kretzoï, 1938) aus den mosbacher Sanden. Mz. Naturu. Arch., Mainz, p. 90-101, 5 fig., 3 tabl.

KRETZOÏ M. 1938 - Die Raubtiere vom Gombaszög nebst einer Übersicht der Gesamtfauna. Ein Beitrag zur Stratigraphie des Altquartärs. Ann. Mus. Hungars; Budapest, $n^{\circ} 31$.

KURTÉN B. 1973 - Pleistocene Jaguars in North America. Commentationes Biologicae, 62. Societas Scientiarum Fennica, Helsinki, 23 p.

TOURNEPICHE J.-F. 1993 - Rapport sur les fouilles effectuées à Artenac (Charente), campagne 1993, $126 \mathrm{p}$. 
TOURNEPICHE J.-F. 1996 - Les grands mammifères pléistocènes de Poitou - Charente. Paléo, nº 8, p. 109 - 141, ill.

\section{NOTES}

1. "les jaguars européens du Villafranchien supérieur et ceux qui se sont succédé de l'Epivillafranchien au Pléistocène moyen inférieur devraient être rapportés à $\mathrm{P}$. onca toscana pour les premiers et $\mathrm{P}$. onca gombaszoegensis pour les seconds"

\section{RÉSUMÉS}

Panthera gombaszoegensis a vécu dès la fin du Pléistocène ancien jusqu'au milieu du Pléistocène moyen. La présence de ce félin en France est peu documentée mais il ne s'agit peut-être que d'un manque de sites. Après un rappel des quelques travaux relatifs à cette espèce, cet article aborde l'étude de la série de vestiges recueillie ces dernières années dans la Grotte XIV (Dordogne) (Fouilles J.-L. Guadelli). On y trouvera la description de ces vestiges et quelques remarques sur la répartition géographique et la phylogénie de ce félin.

Panthera gombaszoegensis lived from the end of the Lower Pleistocene to the middle of Middle Pleistocene. Few remains of this feline have been found in France. However, this pattern may result from very few samples of sites available for this time period. After a review of the literature on this species, this paper deals with the study of remains collected for the last few years in Grotte XIV (Dordogne) (Excavations J.-L. Guadelli). These remains are described and some thoughts are presented on the geographical distribution and on the phylogeny of this feline.

\section{INDEX}

Mots-clés : Panthera gombaszoegensis, Pléistocène ancien, Pléistocène moyen, Villafranchien supérieur, Grotte XIV

Keywords : Panthera gombaszoegensis, Lower Pleistocene, Middle Pleistocene, Upper Villafranchian, Grotte XIV

\section{AUTEUR}

\section{ANNE LANGLOIS}

Institut de Préhistoire et de Géologie du Quaternaire - UMR 5808 du CNRS, Avenue des Facultés, F-33405 Talence cedex 